\title{
Quid des préoccupations pour les primes en Suisse
}

\author{
Nora Willea ${ }^{a}$ Jürg Schlup ${ }^{b}$
}

${ }^{a}$ Dre phil., collaboratrice scientifique du président; ${ }^{b}$ Dr méd., président de la FMH

Dans les statistiques sur les préoccupations de la population suisse, les primes d'assurance-maladie figurent souvent en tête de liste, aux côtés d'autres thèmes comme l'AVS, l'immigration et l'environnement. Mais pourquoi ce sont parfois $9 \%$ et parfois $70 \%$ des sondés qui considèrent les primes comme le problème le plus urgent? Leurs préoccupations générales sont-elles liées au poids des primes? Jetons un coup d'œil sur les chiffres.

Quand il s'agit de déterminer les préoccupations des Suisses, on se tourne généralement vers le «Baromètre des préoccupations». Depuis 1988, cette enquête met en lumière les principaux problèmes aux yeux de la population. La question de la santé et des caisses-maladie est prise en compte depuis 1993 et, y apparaît comme une préoccupation parfois rare, parfois fréquente. Début 2000 , près de deux tiers (64\%) des sondés considéraient cette question comme une préoccupation majeure, dix ans plus tard, cette part a chuté de plus de la moitié à $30 \%$. A partir de 2013 , ce pourcentage est passé pendant quelques années à un peu plus de $20 \%$. Ce chiffre a ensuite doublé pour passer à $41 \%$ au cours des deux der- nières années (fig. 1). Le dernier Baromètre des préoccupations publié en décembre 2019 place la santé et les primes en hausse en deuxième position [1].

La "deuxième préoccupation la plus importante» ne figure pas dans le Top 5 de la majorité

Etre la «deuxième préoccupation la plus importante» ne signifie pas qu'un grand nombre de sondés la citent spontanément. Dans une enquête ouverte, c.à-d. sans proposer le choix de thèmes, la prévoyance vieillesse arrive en tête (41\%), suivie du changement climatique (31\%) et de la question européenne (30\%). La question de la santé et des caisses-maladie apparaît à la deuxième

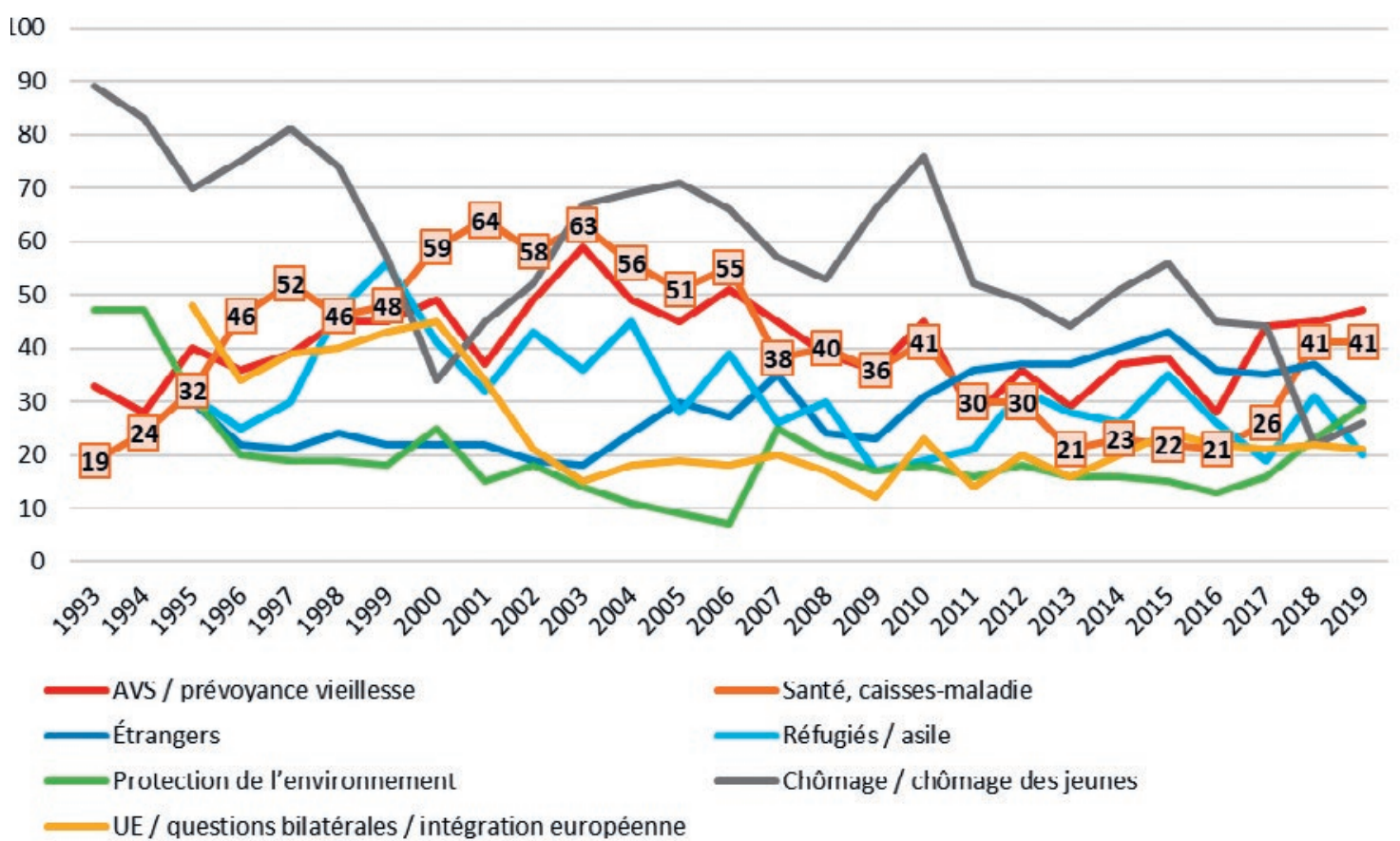

Figure 1: Les points principaux du Baromètre des préoccupations du Credit Suisse 1993-2019 en \% [1]. 
place uniquement si les sondés doivent choisir «les cinq problèmes les plus importants en Suisse» parmi une liste de thèmes: $47 \%$ pour la prévoyance vieillesse, $41 \%$ pour la santé et les caisses-maladie et $30 \%$ pour la question des étrangers. Dans ce contexte, être la «deuxième préoccupation la plus importante» signifie que $41 \%$ de la population la considèrent comme un des cinq problèmes les plus importants en Suisse, alors que 59\% sont d'avis que ce thème n'appartient pas au Top 5 des préoccupations en Suisse [1].

Le résultat est encore différent si on demande lequel des cinq problèmes choisis doit être résolu «en priorité». Dans ce cas, la santé et les caisses-maladie n'occupent que la troisième place avec $9 \%$ de réponse, loin derrière l'AVS / prévoyance vieillesse (16\%) et la protection de l'environnement/le changement climatique (12\%) [1].

\section{Pour $9 \%$, le problème le plus urgent; pour $70 \%$,} un des (nombreux) problèmes pressants

D'autres sondages récents montrent cependant que les primes d'assurance-maladie sont une préoccupation bien plus importante pour la population. Ainsi, le sondage postélectoral de Tamedia réalisé en octobre 2019 a montré que les coûts de la santé étaient la principale inquiétude [2]. Ce sondage proposait 20 thèmes pour lesquels les personnes interrogées pouvaient indiquer par oui ou par non si elles considèrent que les problèmes les plus pressants se situent dans ce domaine. Les coûts de la santé ont obtenu 62\% des réponses (tab. 1). Un mois plus tôt, ce chiffre atteignait même 70\% [3]. De manière générale, chaque sondé a indiqué des "problèmes les plus pressants» dans plusieurs domaines, si bien que dans l'ensemble, la part de oui atteint 508\% [2].

Un autre sondage représentatif du service comparatif moneyland.ch a également identifié les primes d'assurance-maladie comme le "principal souci» [4]. Sur une échelle de 1 («absolument aucun souci») à 10 ("énormément de soucis»), $74 \%$ des sondés ont attribué 7 à 10 points aux primes. Les problèmes environnementaux (70\%), la prévoyance vieillesse en général (66\%), le changement climatique (66\%) et l'AVS (65\%) ont suscité une inquiétude comparable.

Les inquiétudes concernant les primes d'assurance-maladie et les coûts de la santé sont plus marquées quand l'enquête demande d'évaluer individuellement les différents thèmes. Si, par contre, il s'agit de hiérarchiser les priorités, comme dans le Baromètre des préoccupations, ces sujets perdent en importance.

\section{Les primes et la santé préoccupent davantage} les personnes âgées et les Suisses romands

Les résultats de tous les sondages cités jusqu'ici sont cohérents sur un point: les personnes âgées se montrent
Tableau 1: Question «à votre avis, dans quel/s domaine/s se situent les problèmes les plus urgents? (Plusieurs réponses possibles)" du sondage postélectoral de Tamedia, 17 au 20 octobre 2019; $\mathrm{n}=33474$ [2].

\begin{tabular}{ll}
\hline Domaine & Part de «oui» \\
\hline Coûts de la santé & $62 \%$ \\
\hline Prévoyance vieillesse/rentes & $59 \%$ \\
\hline $\begin{array}{l}\text { Changement climatique/destruction } \\
\text { de I'environnement }\end{array}$ & $52 \%$ \\
\hline Rapport avec I'UE & $42 \%$ \\
\hline Migration/immigration & $41 \%$ \\
\hline Asile & $32 \%$ \\
\hline Education & $27 \%$ \\
\hline Situation économique & $21 \%$ \\
\hline Infrastructure de transport & $21 \%$ \\
\hline Criminalité & $19 \%$ \\
\hline Répartition des richesses & $19 \%$ \\
\hline Chômage & $18 \%$ \\
\hline Terrorisme islamiste & $18 \%$ \\
\hline Prix des loyers et de l'immobilier & $18 \%$ \\
\hline Sécurité/armée & $14 \%$ \\
\hline Evolution démographique & $13 \%$ \\
\hline Fondamentalisme religieux & $13 \%$ \\
\hline Situation politique à l'étranger & $12 \%$ \\
\hline Abus de drogues & $4 \%$ \\
\hline Dans un autre domaine & $3 \%$ \\
\hline Aucun/ne sait pas & $1 \%$ \\
\hline
\end{tabular}

davantage préoccupées par le thème de la santé et des caisses-maladie. Ainsi, le sondage moneyland a montré que $52 \%$ des 18 à 25 ans sont préoccupés par ce sujet, alors que ce chiffre grimpe à $82 \%$ chez les 50 à 74 ans [4]. Les régions linguistiques jouent aussi un rôle: $80 \%$ des Romands se soucient des primes de l'assurance obligatoire des soins (AOS), alors que chez les Alémaniques, ce chiffre atteint $70 \%$. En ce qui concerne la santé personnelle, les Romands (79\%) sont nettement plus inquiets que les Alémaniques (45\%).

\section{Les problèmes persistants avec les primes ne} sont pas rares, et pourtant plus rares que jamais Alors que les sondages mentionnés jusqu'ici interrogent d'une manière très générale la population suisse sur ces préoccupations, le moniteur de la santé annuel de gfs.bern se penche concrètement sur l'impact des primes de l'AOS sur la situation financière personnelle. Il passe en revue différentes catégories de dépenses d'un ménage et demande lesquelles constituent «un problème continu ou occasionnel» [5]. Bien que les impôts représentent objectivement des montants plus importants que les primes AOS, ces dernières sont plus souvent considérées comme problématiques. A la fin des années nonante, les personnes indiquant avoir des 


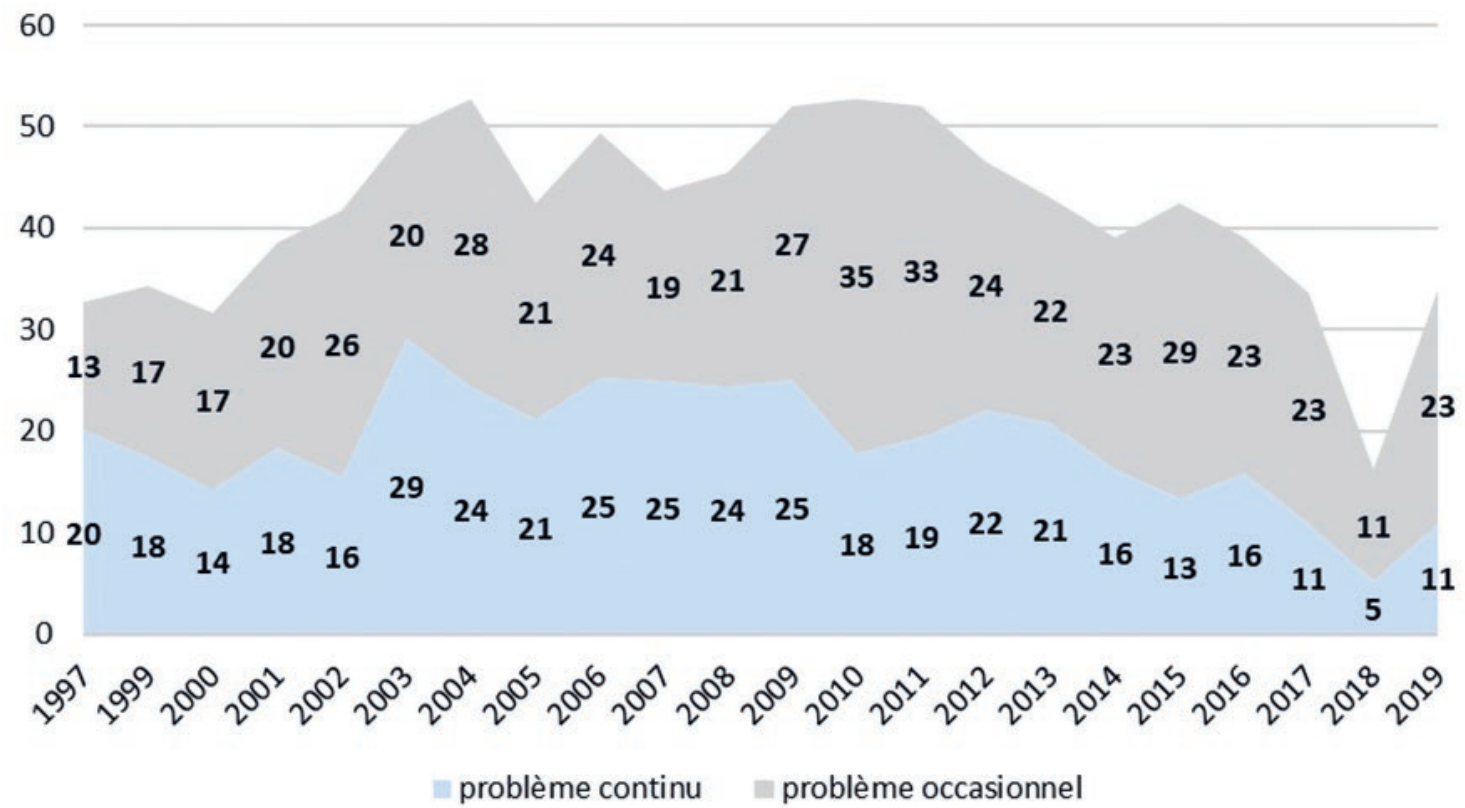

Figure 2: Part de la population (\%) qui considère les primes d'assurance-maladie comme un problème continu ou occasionnel. Résultats du moniteur de la santé gfs 1997 à 2019 [5].

problèmes de primes continus ou occasionnels représentaient environ un tiers, et dès 2003 même la moitié de la population. Depuis 2011, leur part a continuellement baissé. Au cours des trois dernières années, seulement 5 à 11\% des personnes interrogées ont rapporté un problème persistant avec le paiement des primes des valeurs aussi basses n'avaient jamais été atteintes auparavant (fig. 2).

\section{Les préoccupations générales ne reflètent pas forcément les problèmes personnels}

La fréquence à laquelle les primes sont perçues comme problématiques pour certaines personnes semble cependant peu liée à la perception générale de la question (fig. 3). Ainsi, entre 2003 et 2011, une part plus ou moins constante d'environ $50 \%$ des personnes interrogées a mentionné des problèmes occasionnels en rapport avec les primes de l'AOS. Pourtant, la question de la santé et des caisses-maladie a fortement perdu en importance sur la même période dans le Baromètre des préoccupations. La part de personnes qualifiant cette question de préoccupation importante est passée de 63 à 30\%. Dans les années 2013 à 2016, elle a atteint les valeurs les plus basses depuis 20 ans même si, parallèlement, une part constante d'environ $40 \%$ des sondés indiquaient des problèmes au moins occasionnels avec le paiement des primes. En 2018 et 2019, ce pourcentage a de nouveau doublé, tandis que le nombre de personnes qui considéraient les primes comme une charge pour leur budget a diminué. Lors- qu'en 2019, environ 70\% des personnes interrogées par Tamedia et moneyland considéraient les primes comme une des préoccupations les plus urgentes, $65 \%$ des sondés répondaient en même temps à gfs.bern ne même pas avoir occasionnellement des problèmes avec le paiement des primes.

\section{Primes: aucun lien clair entre inquiétudes et évolution}

L'évolution objective des primes ne semble pas non plus se répercuter directement dans les statistiques relatives aux préoccupations (fig. 3). Entre 1998 et 2017, la part des primes dans le budget des ménages a continuellement augmenté de 4,3 à 6,5\% [6]. Les inquiétudes générales et personnelles liées aux primes n'ont cependant pas connu d'augmentation constante, mais étaient parfois plus rares et parfois plus fréquentes. Dans les années 2000 et 2001 , lorsque la prime moyenne a augmenté de 3,2 et 3,6\%, le Baromètre des préoccupations affichait environ $60 \%$, et ce pourcentage est resté le même les années suivantes, alors que l'augmentation des primes a été nettement plus marquée (jusqu'à 9\%). En 2010, la prime moyenne a augmenté de près de $9 \%$ et le baromètre affichait des inquiétudes nettement moins marquées (environ 40\%) mais égales à celles de 2008, alors que la prime moyenne avait même baissé cette année-là. Evidemment, on pourrait interpréter la baisse à long terme du Baromètre des préoccupations comme une réaction aux hausses de primes moins importantes. Cela serait toutefois en contradiction avec la forte aug- 


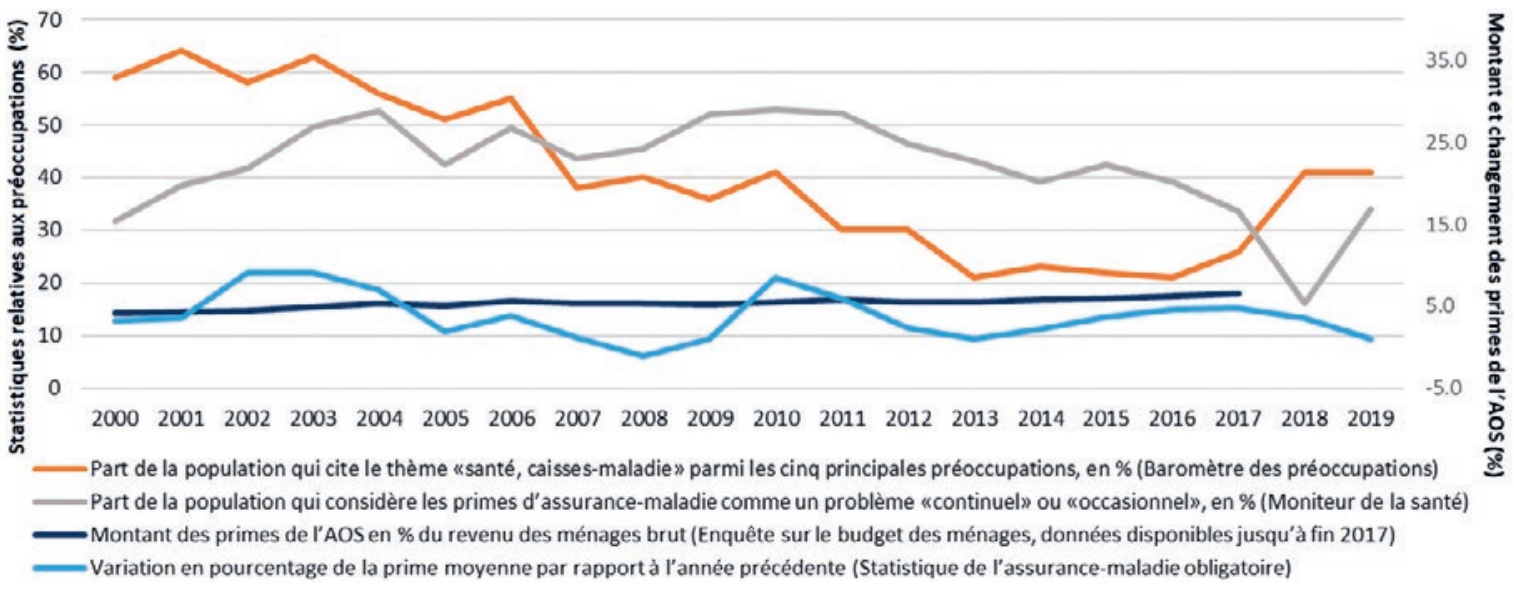

Figure 3: Evolution des primes comparativement à l'évolution des préoccupations (données de [1, 5, 9, 10]).

mentation des valeurs du Baromètre des préoccupations à nouveau enregistrées en 2018 et 2019.

\section{Les inquiétudes reflètent de réels problèmes et bien plus encore}

Un regard sur les statistiques montre que l'ampleur des inquiétudes liées aux primes ne dépend pas seulement de la question de savoir qui est interrogé mais aussi quelles questions sont posées et comment. Si on demande simplement dans quelle mesure les primes de l'AOS sont un motif d'inquiétude, on obtiendra plusieurs majorités indiquant un problème. S'il s'agit par contre de hiérarchiser les thèmes présentés, le nombre de personnes citant les primes comme inquiétude importante sera moins élevé. Enfin, si on pose des questions ouvertes sur les principaux problèmes, le thème "santé et caisses-maladie» ne joue pas un rôle déterminant.

Une comparaison entre différentes séries chronologiques montre que l'évolution de la fréquence des préoccupations ne peut pas ou pas uniquement être expliquée par l'évolution réelle des primes. Pourtant, les inquiétudes concernant les primes de l'AOS sont probablement motivées par des problèmes réels dans les ménages du quintile financièrement le plus faible. Dans ces ménages, le poids des primes de l'AOS est élevé [7] et a sensiblement augmenté [6]. En même temps, les chiffres montrent aussi que les personnes qui n'ont pas de difficultés à payer leurs primes d'assurance-maladie les considèrent quand même comme un sujet de préoccupation. Les métaphores menaçantes telles que l'«explosion des primes» et parfois même des affirmations erronées (pour des exemples, cf. [6] et [8]), véhiculées depuis de nombreuses années dans le débat public, n'y sont peut-être pas étrangères. A cela s'ajoute que même les développements positifs sont interprétés négativement comme des "phases de répit». Le tableau complet des chiffres laisse donc entrevoir que les statistiques relatives aux préoccupations re- flètent d'une part les inquiétudes fondées sur des problèmes réels, mais englobent aussi des soucis suscités par un débat public déstabilisant. Par ailleurs, les chiffres montrent que les inquiétudes se relativisent vite, dès qu'il s'agit de définir des priorités. Enfin, aucun des chiffres quantifiant les préoccupations ne tient compte du conflit suscité par les objectifs visant, d'une part, les primes basses et, d'autre part, un accès à une médecine de qualité élevée. Les statistiques ne font donc que confirmer la nécessité de trouver de bons compromis pour résoudre ce conflit, sans pour autant dire à quoi ils devraient ressembler.

\section{Références}

1 Golder L, et al. Credit Suisse Sorgenbarometer 2019. Aufgabe nicht erfüllt? Reformstau, Führungslosigkeit und die Erwartung einer Wirtschaftskrise hinterlassen Spuren. gfs.bern, décembre 2019.

2 Leeman L, et al. Tamedia-Nachwahlumfrage 2019. LeeWas GmbH und Tamedia AG, 2019.

3 Leeman L, et al. Tamedia-Wahlumfrage 2019, 6. Welle, septembre 2019. LeeWas GmbH und Tamedia AG, 2019.

4 Moneyland.ch. Baromètre des préoccupations: les plus grands soucis des Suisses, article en ligne du 4.9.2019; URL: https://www.moneyland.ch/fr/barometre-preoccupations-soucis-suisse-2019

5 Bieri U, et al. L'essentiel en bref sur le Moniteur de la santé 2019. La qualité avant les coûts, moins de disposition à renoncer. gfs.bern. Interpharma, 2019, Bâle.

6 Wille N, Schlup J. Comment évolue la charge des primes pour les ménages? Bull Med Suisses. 2020;101(38):1075-80.

7 Wille N, Schlup J. Que dépensons-nous pour les primes d'assurance-maladie? Bull Med Suisses. 2020;101(36):1057-60.

8 Wille N, Schlup J. Le poids des primes dans le budget des ménages un bilan chiffré. Caisses-maladie: qui paie combien en Suisse? Bull Med Suisses. 2019;100(37):1212-5.

9 Revenus et dépenses de tous les ménages par année, T20.02.01.00.01; ou évolution des revenus et dépenses des ménages, T20.2.1.1; https://www.bfs.admin.ch/bfs/fr/home/statistiques/situation-economique-sociale-population/revenus-consommation-et-fortune/budget-des-menages. assetdetail.10867220.html

10 Office fédéral de la santé publique, T 1.01 Assurance-maladie obligatoire dès 1996: principaux indicateurs; URL: https://www.bag. admin.ch/bag/fr/home/zahlen-und-statistiken/statistiken-zurkrankenversicherung/statistik-der-obligatorischen-krankenversicherung.html 